Vietnam Journal of Mechanics, VAST, Vol, 27, No. 1 (2005), pp. $13-22$

\title{
THE INFLUENCE OF THE MECHANICAL FATIGUE ON THE ENERGY LOSS OF FERROMAGNETIC STEEL BY MAGNETIC METHOD
}

\author{
Van HuU Thinh ${ }^{1}$, NguYen Xuan Hung ${ }^{2}$ \\ ${ }^{1}$ University of Technical Education HCM city \\ ${ }^{2}$ Institute of Applied Mechanics HCM city
}

\begin{abstract}
In this paper we present some experimental results of the influence of the fatigue on the energy loss due to hysteresis of the rotating sample made from ferromagnetic steel in the fatigue testing. By measuring and calculating the energy loss per cycle due to hysteresis, we discover the energy loss increase depending on number of fatigue cycles. The results are used for evaluating the fatigue state, the working life, and technical diagnosing for rotating samples.
\end{abstract}

\section{INTRODUCTION}

Most machine parts are subjected to variation in applied loads, causing fluctuation in stresses in the parts. If the fluctuating stresses are of sufficient magnitude, even though the maximum applied stress may be considerably less than the static strength of the material, failure may occur when the stress is repeated a sufficient number of times. This failure is called the fatigue failure. The important effect of the fatigue in metals is to change the mechanical properties of metal as shown in [1].

It has been recognized for sometime that the magnetic characteristics of a material are related to its composition and it $\mathrm{s}$ mechanical properties. The object of this work is to establish the influence of the mechanical fatigue on the magnetic properties of ferromagnetic sample used. There are many methods of magnetic analysis that are used to measure the magnetic properties. It has been found that alternating current method have several advantages and are most suitable for material of uniform cross section. In this method the sample under observation forms the core of a coil or a solenoid. The various characteristic of the core cause the shifts in the phase angle between the current and the voltage of the induced current and give the hysteresis loop, the energy loss per cycle. By measuring the energy loss per cycle due to hysteresis, we can evaluate the change in magnetic characteristics of the sample, and make predictions on its working life.

In order to find the influence of the mechanical fatigue on the magnetic properties of AISI 1045 steel sample we performed several experiments on the rotating beam testing machine. The experimental researches show that the fatigue makes the change in the energy loss per cycle due to hysteresis.

\section{BASIS OF THEORY}

\subsection{Ampere's law}

One of the basic theorems in the electromagnetic is the Ampere's law which relates the magnetic produced by an electric current to the current passing through a conductor. 
Ampere's law states that the line integral of the magnetic field $H$ taken around a closed path is equal to the total current enclosed by the path:

$$
\oint H d l=\sum i \text {. }
$$

For a uniform field, $H$ is a constant and we have:

$$
H l=\sum i .
$$

Or if $H$ is constant over sections, with different section having different $H$, then:

$$
\sum H l=\sum i .
$$

\subsection{Magneto-motive force ( $\mathrm{mmf}$ )}

Magneto-motive force is the flux producing ability of an electric current in a magnetic circuit:

$$
m m f \Im=\sum i .
$$

Consider a coil having $N$ turns. It will link the flux path with each turn, so that total current linking with the flux would be

$$
\sum i=N i .
$$

Thus from Ampere's law, the mmf produced by the coil of $N$ turns would be

$$
N i=H l .
$$

\subsection{Magnetic flux density}

The magnetic flux $H$ gives rise to a magnetic flux $\phi$, which has a magnetic flux density $B$ for a given area $A$. The relationship between $B$ and $H$ is given the permeability of the medium $\mu$.

where

$$
B=\mu H,
$$

$$
\mu=\mu_{0} \mu_{r},
$$

$\mu_{r}$ is the relative permeability which can vary from about 2.000 to about 100.000 dependant on the material.

$\mu_{0}=4 \pi \times 10^{-7} \mathrm{H} / \mathrm{m}$ : permeability of air is generally taken to be equal to that of free space in practice.

$$
\phi=B A .
$$

Unit of permeability is henry per meter $(\mathrm{H} / \mathrm{m})$.

Unit of magnetic flux density is tesla $(T)$.

Unit of magnetic flux is weber $(\mathrm{Wb})$ 
Unit of magnetic field is ampere per meter $(\mathrm{A} / \mathrm{m})$.

\subsection{Reluctance of a magnetic path}

A magnetic material presents a reluctance $S$ to the flow of magnetic flux when an mmf is applied to the magnetic circuit.

Thus mmf $=$ Reluctance $x$ flux .

$$
\Im=S . \phi .
$$

For a uniform field: $\Im=N i=H l$.

From (2.7) and (2.8), we have: $\phi=B A=\mu H A$.

From (2.10), we have: $H l=S \mu H A$.

$$
S=\frac{l}{\mu A},
$$

where $l$ : length, $A$ : area of the cross section.

Unit of magnetic reluctance is $1 /$ henry $\left(H^{-1}\right)$.

Magnetic permeance $\Lambda$ is the inverse of the magnetic reluctance. Thus

$$
\Lambda=\frac{1}{s}=\frac{\mu A}{l} .
$$

Unit of magnetic permeance is henry $(\mathrm{H})$.

\subsection{Self inductance}

While the reluctance is a property of the magnetic circuit, the corresponding quantity in the electrical circuit is the inductance.

From the Faraday's law of electromagnetic induction, induced emf:

$$
\begin{aligned}
& e=N \frac{d \phi}{d t}=L \frac{d i}{d t}, \\
& N \phi=L i, \\
& L=\frac{N \phi}{i} .
\end{aligned}
$$

The self inductance $L$ of a winding is the flux linkage produced in the same winding due to unit current flowing through it. For a coil of $N$ turns, if the flux in the magnetic circuit is $\phi$. The flux linkage with the coil would be $N \phi$.

Also since $N i=S \phi$

$$
L=\frac{N^{2}}{S}=\frac{N^{2} \mu A}{l} .
$$

Thus the inductance of a coil of $N$ turns can be determined from the magnetic circuit.

Note that the inductance of a coil is directly proportional to the permeability of the medium inside the core of the coil. By using a ferromagnetic material such as iron as the core, the inductance can be increased significantly given the large relative permeability of a ferromagnetic material. 


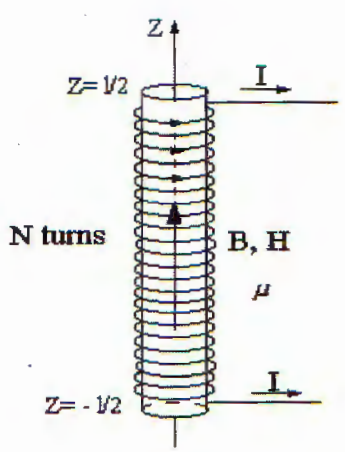

Fig. 1. The solenoid and its core

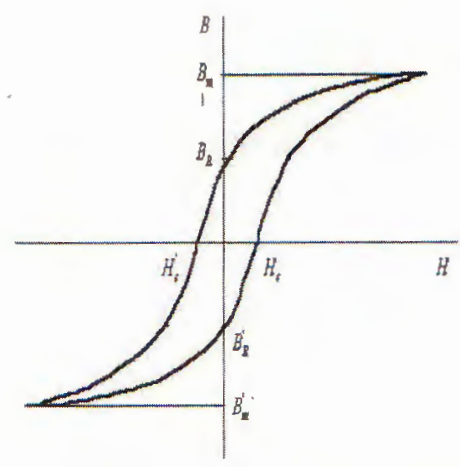

Fig. 2. The hysteresis loop

\subsection{Energy loss due to hysteresis}

For the solenoid with non-ferromagnetic core, the electrical energy converted to energy in the magnetic field during the rise of the current is all reconverted into electric energy when the current decrease to zero. If the core material is ferromagnetic, however, some of the magnetic energy is converted irreversibly into heat as a result of the hysteresis . behavior. The amount of electrical energy in one hysteresis cycle, which is converted to magnetic energy but is not reconverted to electrical energy, is called energy loss in one cycle due to hysteresis. Let us now compute this amount of this electrical energy.

Let $e$ present the instantaneous emf of self-induction set up in the solenoid, as shown in Fig. 1, due to the rate of change of $i$, where $i$ is the instantaneous current flowing in the solenoid. Also, let dW represent the element of energy which is transferred from the electric circuit to the magnetic field of the solenoid in an element of time $d t$. When $i$ is increasing in magnitude, $e$ is in an opposite sense to that of $i$, and electrical energy is being converted into magnetic energy so $d W$ is positive during an increase in the magnitude of $i$. When $i$ is decreasing in the magnitude, $e$ is the same sense as $i$, and magnetic energy is being converted into electrical energy so $\mathrm{dW}$ is negative during a decrease in the magnitude of $i$. For any infinitesimal length of time $d t$, we have

$$
d W=i e d t
$$

But, from Faraday's law of electromagnetic induction (13),

$$
e=N \frac{d \phi}{d t}=N A \frac{d B}{d t}
$$

where $\phi$ is the instantaneous value of the total magnetic flux through the core, $B$ is the instantaneous magnetic flux density, $A$ is the area of cross section of the core.

Put (2.16) into (2.15) and we have

$$
d W=N A i d B \text {. }
$$

From (6), Ni $=H i$. Put this value of $N i$ into (2.17) and

$$
d W=A l H d B
$$


However, $A l=v$, where $v$ is the volume of the core, so

$$
d W=v H d B
$$

Let $W$ represent the net energy input to the magnetic field during one cycle, i.e, $W$ is the energy is not recovered by the electric circuit. Then

$$
W=v \oint H d B .
$$

Break the integral of (2.19) into the following parts for evaluation

$$
\oint H d B=\int_{B_{m}}^{B_{r}} H d B+\int_{B_{r}}^{B_{m}^{\prime}} H d B+\int_{B_{m}^{\prime}}^{B_{r}^{\prime}} H d B+\int_{B_{r}^{\prime}}^{B_{m}} H d B .
$$

The energy represented by the area enclosed in the hysteresis loop shown in Fig. 2 is fed from the electric circuit into the magnetic but is not returned to the electric circuit, thus (2.19) becomes.

$$
W=v \text {.(area enclosed in the hysteresis loop). }
$$

If the frequency of the alternating current flowing in the coil is $f$, the core material goes through the hysteresis cycle of changes $f$ times per second and the power loss due to hysteresis is

$$
P=v f(\text { area enclosed in the hysteresis loop). }
$$

Thus, for evaluation of the energy loss $W$ or the power loss $P$ due to hysteresis in the core of a solenoid or a transformer, we must determine the area enclosed in the hysteresis loop. In order to calculate the area enclosed in the hysteresis loop, we can perform as the following procedure:

- Using measuring device system, we have the hysteresis loop for one cycle.

- Determining the area enclosed in the hysteresis loop in terms of square millimeters or some similar area unit.

- Multiply the area by the scale factor, to determine the area in unit of $B$ times $H$.

- Substitute this area into (2.21) or (2.22), thereby determine the energy loss $W$ or the power loss $P$ due to hysteresis.

In order to research the influence of the mechanical fatigue on the energy loss per cycle of ferromagnetic samples by the magnetic method, we performed the following experiments. 


\section{EXPERIMENTAL METHODS}

\subsection{Experiment set up}

The purpose of the experiment is to detect the change in the energy loss per cycle of the ferromagnetic sample in the rotating beam fatigue test.

The samples were made from AISI 1045 steel with the following chemical composition $(\%): C=0.45, S i=0.30, M n=0.70, S=0.04$. The mechanical parameter of this steel is tensile strength $=650 \mathrm{~N} / \mathrm{mm}^{2}$. The shapes and dimensions of the samples are given in Fig. 3. The fatigue testing machine is shown in Fig. 4. The samples was subjected to the magnetic field with the frequency $f=50 \mathrm{~Hz}$, and the voltage $u=6 \rightarrow 24 \mathrm{~V}$ created by $\mathrm{AC}$ supply and a transformer shown in Fig. 5.

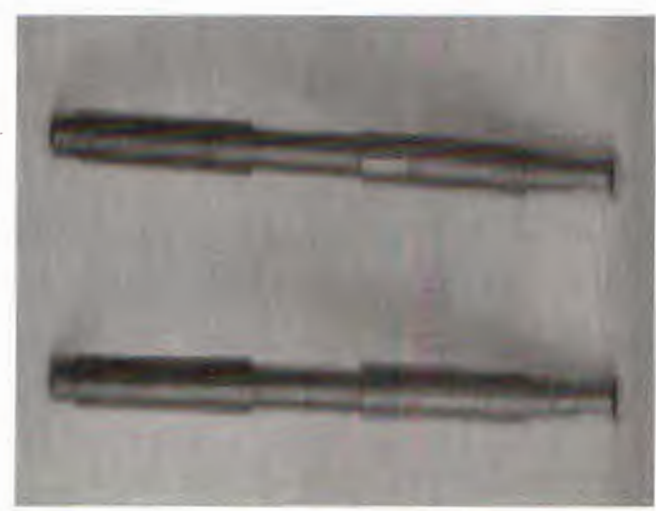

Fig. 3. The testing sample

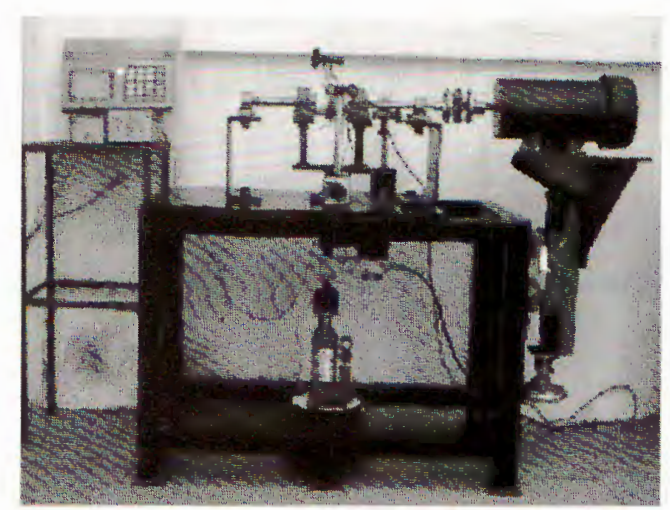

Fig. 4. The fatigue testing machine

The experiment conditions for each sample are constant such as the applied force, the speed, the frequency and the voltage of AC supply, and the measurement method. The sample time is $10^{5}$ revolutions of the rotating sample. The schematic of measurement device system is shown in Fig. 6 . It consists of the following devices:

- Load cell + Digital force indicator.

- A hydraulic jack to 2 tons.

- A coil with 10.000 turns.

- An electric circuit.

- A revolution counter set.

- Amplifier and Filter set.

- FFT (Fast Fourier Transformer).

- A computer. 


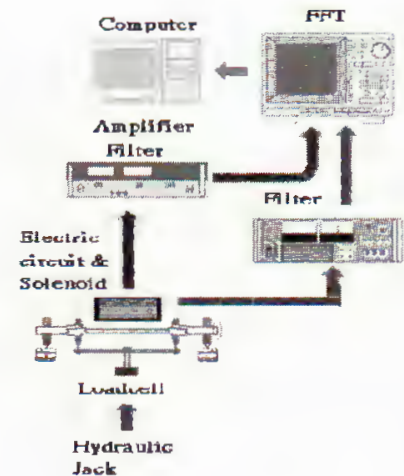

Fig. 5. Schematic of measuring device system

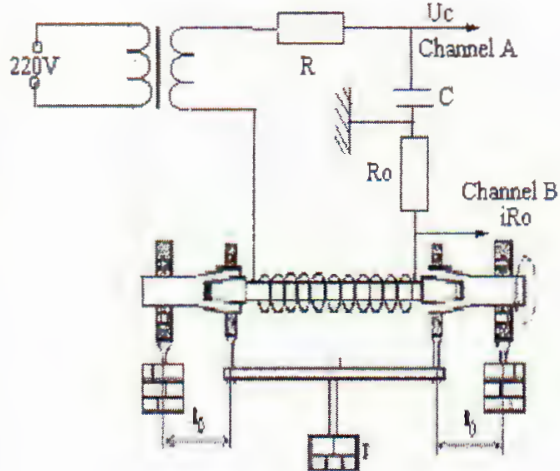

Fig.6. The electric circuit and the testing sample

\subsection{The measurement method}

The measured data were displayed on the FFT:

- Channel A: the induced emf signal $u_{c}(t)$ as shown in Fig7

The induced emf: $u_{c}(t)=\frac{1}{R C} \int e d t=\frac{N A}{R C} B(t)$.

Thus $u_{c}(t)$ is proportional to the magnetic flux density $B(t)$.

- Channel $B$ : the $i R_{0}$ voltage signal as shown in Fig. 8.

This is the voltage through the resistor $R_{0}$. This voltage is proportional to the induced current. Thus it is also proportional to the magnetic field $H(t)$.

- Combine two signals, we obtain the hysteresis loop on the FFT as shown in Fig.9.

- The measured data are transmitted to the computer.

- Using the software as shown in Fig.9, the area of the hysteresis loop is determined.

Note that the hysteresis loop area = the energy loss per cycle of the sample.

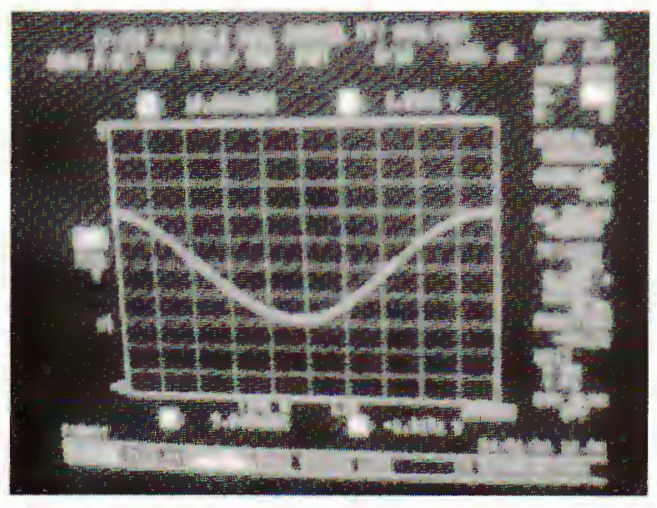

Fig. 7. The induced emf signal

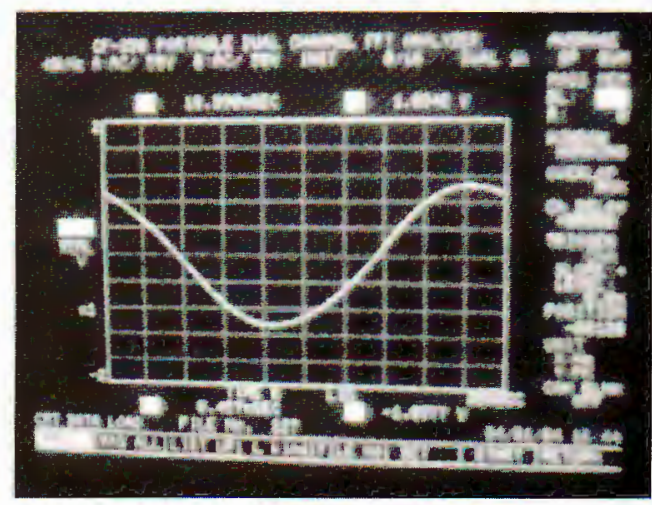

Fig. 8. The $i R_{0}$ voltage signal 


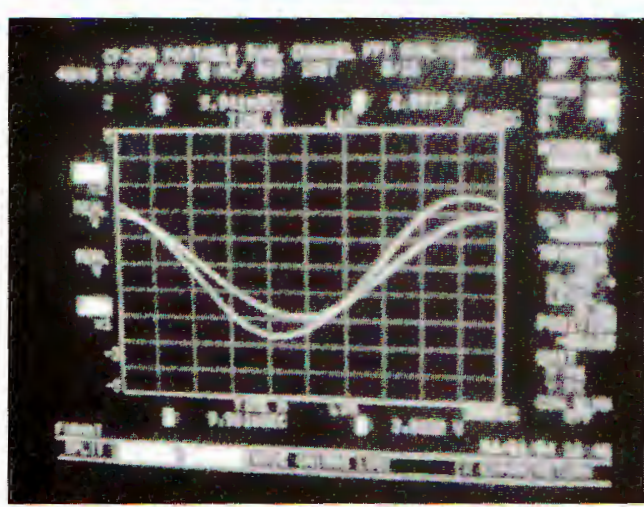

Fig. 9. The shift in phase angle

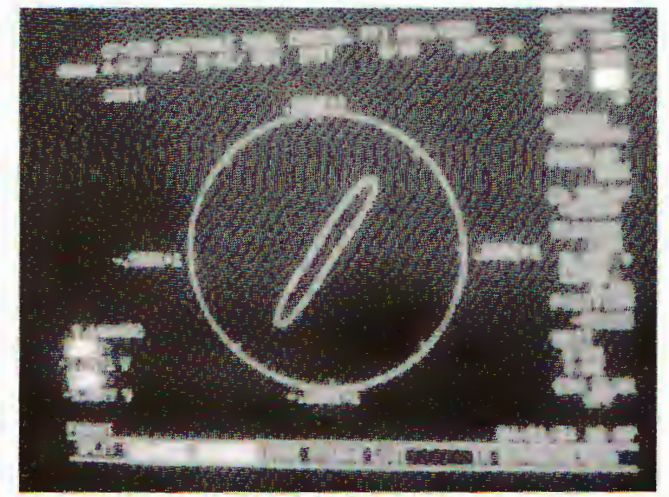

Fig. 10. The hysteresis loop

Between two signals

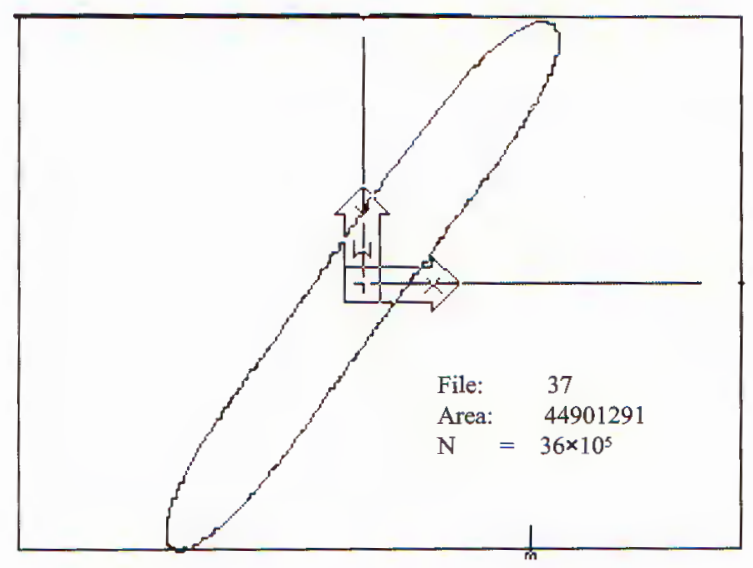

Fig. 11. The software for calculating the area of the hysteresis loop

\subsection{Experimental results}

The experimental results were stored in two ways:

* FFT: the measured data from channel $A \& B$ were stored in the floppy disk $1.4 \mathrm{MB}$, and transmitted to the computer.

* Computer: the data were stored, constructed, and calculated the hysteresis loop area responding to the sample time.

In order to evaluate the change in the energy loss per cycle of the sample versus the number of cycles, we constructed the plot that shows the relative energy loss per cycle $\Delta D$ versus fatigue accumulation $=\sum_{i=1}^{m} \frac{n_{i}}{N_{i}}$.

Where $\Delta D=D_{I}-D_{0}$

$D_{I}$ : energy loss per cycle spent number of cycle $n_{i}$.

$D_{0}$ : energy loss per cycle spent number of cycle $n_{0}=0$.

The way in which the energy loss for one cycle changes depending on the number of fatigue cycles was performed for several experiments with various the applied force and 
speed conditions.

For illustration we present one of the experimental results as shown in Figures 11 13. This sample was performed in the experimental conditions: applied force $=20 \mathrm{KG}$, speed $=1500 \mathrm{rpm}$, channel A:ac $/ 5 \mathrm{v}$, channel B:ac $/ 2 \mathrm{v}$, fatigue failure cycles $=3.5$ million revolutions. The Figures present the change in the shape and the area of the hysteresis loop versus number of fatigue cycles of this sample.

The experimental results reveal that the influence of the mechanical fatigue makes increase the energy loss due to hysteresis of the ferromagnetic sample made from AISI 1045 steel.

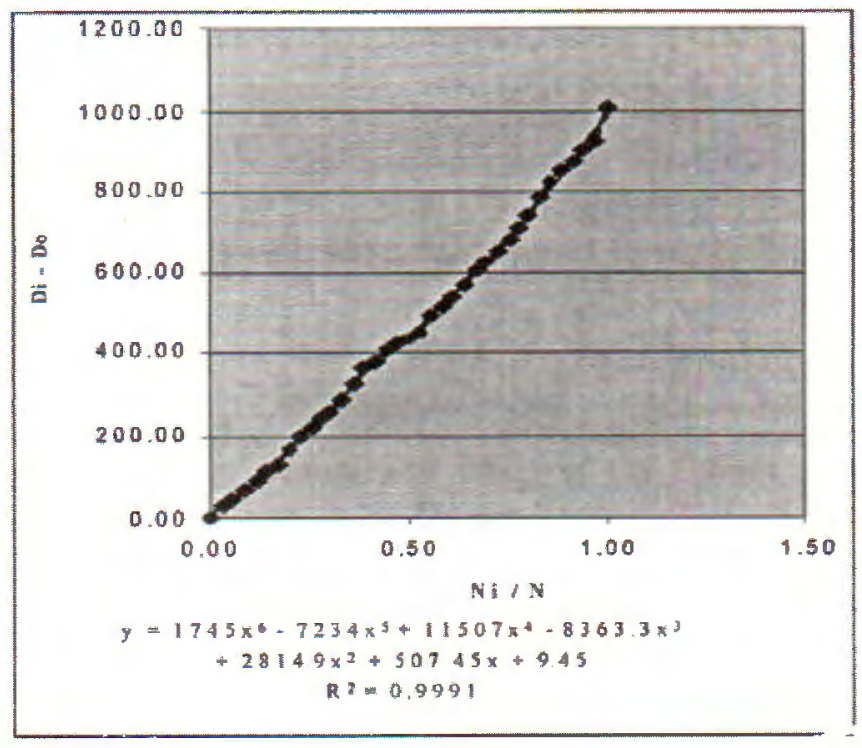

Fig. 12. The change in the energy loss per cycle versus the number of cycles

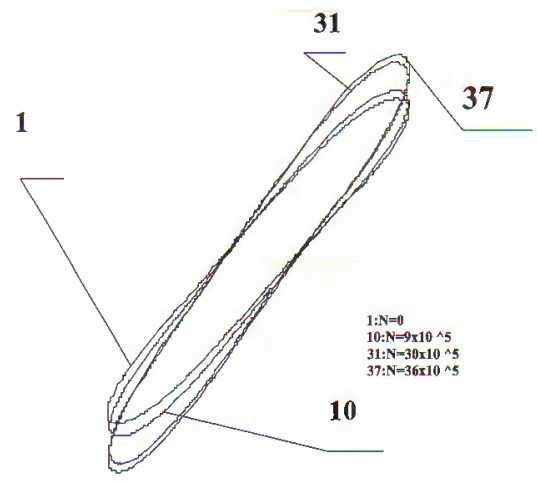

Fig. 13. The change in the hysteresis loop versus the number of cycles

Energy loss due to hysteresis is work, which is done in aligning the Amperian current loops and domain in the core (sample) of the solenoid. In the core there is a kind of internal friction against the alignment. From the research results in [1] when the sample 
rotates there is an energy dissipation due to internal friction, as the result of the relative motion between particles of the steel during deformation. This energy dissipated increase depending on number of fatigue cycles. This leads to increase the energy loss due to hysteresis is as the experimental results above.

\section{CONCLUSIONS}

This work leads to the following conclusions:

- The change in mechanical properties of the sample in the fatigue test has connection with its magnetic properties.

- The energy loss per cycle due to hysteresis increase depending on number of fatigue cycles.

- The results are used for evaluation the fatigue state, and diagnosing the working condition of the rotating samples.

This publication is completed with financial support from the National Basic Research Program in Natural Sciences.

\section{REFERENCES}

1. Nguyen Xuan Hung, Van Huu Thinh. The Effect of the Fatigue on elastic Modulus and damping Coefficient in the Rotating Beam Fatigue Testing, ISDC, Hanoi, 2003.

2. Nguyen Xuan Hung, Dynamics of Structures and compliant Mechanisms, 2003.

3. Nguyen Xuan Hung K Miya, M. Yamamoto Editors. Applied Electromagnetism and Mechanics, Proceedings of the First Vietnam-Japan Symposium on Advances Electromagnetics and Mechanics, January 19-21,1998, Hochiminh City, Vietnam.

4. John D. Kraus, Electromagnetics, McGraw-Hill international Editions, 1992.

5. Ralph P. Winch, Electricity and Magnetism, Prentice Hall, Inc. 1957.

6. Lucas J. R., Electromagnetic Theory, 2001.

7. Chifan S., Grimberg R. et al., Evaluation of Fatigue State of ferromagnetic Steels by Magnetic Method, Roma $200015^{\text {th }}$ WCNDT.

8. Jean. Marie Brebec, Philippe Deneve et al., Electromagnetisme, Translated into Vietnamese by Le Bang Suong, Education Publishing House, 2001.

Received February 1, 2004

\section{ÅNH HƯởNG HIỆN TƯợNG MÒI ĐẾN TỔN HAO NĂNG LƯợNG CỦA MẪU SẮT TƯ BẰNG PHƯƠNG PHÁP PHÂN TÍCH TỬ}

Bài báo trình bày kết quả thực nghiệm về ảnh hường của hiện tượng mỏi đến tồn hao năng lượng do từ trễ của mẫu chễ tạo bằng sắt từ trong thí nghiệm mơi. Bằng cách đo thực nghiệm và tính toán bằng phần mềm, đã phát hiện qui luật tổn hao năng lượng do từ trễ phụ thuộc vào số chu kỳ làm việc. Ta có thể áp dụng kết quả nghiên cứu này để đánh giá trạng thái mơi, tuổi thọ làm việc và chẩn đoán kỹ thuật đối với mầu hay trục máy.

E-mail: ptcnm@hcm.vnn.vn 